\title{
El método de casos como alternativa pedagógica para la enseñanza de la bibliotecología y las ciencias de la información
}

\author{
Alonso Estrada Cuzcano \\ Karen Lizeth Alfaro Mendives *
}

Artículo recibido:

24 de noviembre de 2013.

Artículo aceptado:

5 de diciembre de 2013.

\section{Resumen}

El presente estudio pretende demostrar la influencia significativa del método de casos como técnica y metodología válida en la enseñanza de los cursos impartidos en la Escuela de Bibliotecología y Ciencias de la Información. Se presentan como experiencia los resultados obtenidos con esta metodología en el curso de Administración de Unidades de Información. Se puede concluir que el método de casos es efectivo, pues no sólo mejora significativamente los resultados cuantitativos del aprendizaje, sino también, desde el punto de vista cualitativo, es una experiencia gratificante, pues se genera mayor comunicación con el estudiante, quien presenta una actitud más crítica, se involucra

* Ambos autores pertenecen a la Universidad Nacional Mayor de San Marcos, Perú. (Alonso: mestradac@unmsm.edu.pe); (Karen: karen_alfarom@yahoo.es)

INVESTIGACIÓN BIBLIOTECOLÓGICA, Vol. 29, Núm.65, enero/abril, 2015, México, ISSN:
0187-358X. pp. 195-212 
e interioriza los temas; y sobretodo es una metodología dinámica que permite un clima óptimo para el aprendizaje. Asimismo, se establece en el artículo una aproximación histórica, donde las definiciones y características fundamentales, los pasos a seguir para el desarrollo y el estudio de casos permiten la fundamentación teórica y la contextualización de la investigación.

Palabras clave: Método de casos; Enseñanza universitaria pedagógica; Bibliotecología.

\section{Abstract}

Case method as pedagogical alternative for teaching Library and Information Sciences

Alonso Estrada-Cuzcano and Karen Lizeth Alfaro-Mendives

Based on an examination of the use of case method in Information Unit Management courses, this study aims to demonstrate the significant influence of this approach as a valid methodology for teaching courses in the School of Library and Information Science. Researchers conclude that the case method is effective: it not only significantly improves the quantitative results of learning; but also, from a qualitative standpoint, enriches the teaching/learning experience through improved communication with students, while encouraging critical thinking and better overall engagement with curricular objectives. The paper also offers a historical overview of the case method, key definitions and features, steps for implementation and several case studies to support the theoretical context and foundations of the research.

Keywords: Case Method; Academic Teaching; Librarianship.

\section{INTRODUCCIÓN}


diferencias y diversos puntos de vista y requiere cumplir con algunas condiciones relacionadas con la forma y el estilo necesarios para su eficaz desarrollo en el aula. El método es adecuado para la enseñanza universitaria porque no está basado sólo en la intervención o conocimientos del docente sino fundamentalmente en la participación activa del estudiante, quien identifica, analiza, sistematiza, evalúa, propone y decide cómo resolver las situaciones o problemas planteados en el caso.

Este método de enseñanza no sólo comprende la recepción de contenidos, sino también la asimilación personal y ahí reside su fuerza radical (Bañares Parera, 2011). Los estudiantes acumulan experiencias en muchas discusiones y los casos se prueban constantemente; en consecuencia, no es posible realizar una evaluación (examen) de forma absoluta o única (Ardalan, 2013).

El método de casos es interactivo (estudiante-docente) y los conocimientos se construyen paso a paso; podría considerarse como una técnica de simulación en la clase, aunque previamente el docente debe planificar el método según las características del entorno, los destinatarios y la complejidad del asunto tratado.

Por supuesto que este método también es usado (o debería usarse) en el ámbito de la bibliotecología y las ciencias de la información, cuyo carácter es eminentemente interdisciplinario. También es factible utilizar el método de casos en múltiples materias impartidas en la enseñanza de la bibliotecología: gestión de unidades de información, ética y deontología de la información, derecho a la información, políticas de información, estudios de usuarios, entre otros. Como ejemplo, se puede citar a la Library Journal publicada por la American Library Association; esta revista tiene una sección denominada "How do you manage" donde se analizan y discuten casos controversiales recogidos del propio colectivo profesional.

Este método es también utilizado con frecuencia para resolver los dilemas éticos existentes. Hannabuss (1996) indica que es un método válido en la enseñanza de la ética y que juega un rol fundamental entre aquellos estudiantes que tienen diversos niveles intelectuales, morales y profesionales respecto a asuntos de relevancia ética.

A pesar de la aplicación del método de casos en la enseñanza universitaria desde hace mucho tiempo, no hay una extensa sistematización o recopilación de experiencias, ni existe la literatura suficiente (numerosa y actualizada) para orientar de forma idónea a los docentes que desean aplicarlo.

Se puede hacer un recuento histórico a partir del texto de Garvin (2003); en el artículo "Making the Case" señala que el pionero del método fue Christopher Columbus Langdell quien asistió a la Harvard Law School entre 1851 
y 1854, donde se desenvolvió como asistente de investigación y bibliotecario; allí demuestra rápidamente su destreza principal: la investigación y los resúmenes. En 1870, el presidente de Harvard, Charles William Eliot, recomienda a Langdell para difundir y enseñar la aplicación del método de casos en la universidad.

Langdell partió del supuesto de que la mejor manera de enseñar leyes era mostrando casos en lugar de leer los libros de texto, y luego de algunas resistencias iniciales el método fue asumido por otras seis escuelas de leyes en 1895. Posteriormente, en 1921, la Harvard Bussiness School adoptó el método y se le denominó como aún se le conoce en la actualidad: "Método de Casos”. El mismo Garvin (2003) afirma que a partir de 1985 la Harvard Medical School utiliza el método de casos. La historia podría resumirse así: "Hace cien años aproximadamente, Harvard Business School inició un nuevo enfoque en la enseñanza llamado el método de caso. Diseñado a partir del uso de casos en la Escuela de Derecho, la Facultad de Negocios comenzó a usar historias reales de prácticas de negocios para instruir a los estudiantes. El método del caso se ha transformado significativamente desde entonces" (Herreid, 2011: 31).

\section{CARACTERÍsticas DEL MÉTODO DE CASOS}

El método de casos es una técnica (y metodología) educacional que pone a consideración del estudiante situaciones y problemas verdaderos que conducen a la presentación de alternativas de solución o a finalmente resolverlos; podría decirse que el método es un tipo de discusión interactiva que está estructurado sobre la base del estudio de casos y el cual es referido comúnmente con el término caso.

Caso proviene etimológicamente del latín casus, en el Diccionario de la Real Academia Española (RAE, 2013) aparecen varias acepciones de la palabra, pero sólo tomaremos algunas definiciones que se acercan más a nuestro punto de vista: "suceso, acontecimiento", "casualidad, acaso", "lance, ocasión o coyuntura" y "asunto de que se trata o que se propone para consultar a alguno y pedirle su dictamen”. Creemos que esta última acepción es más cercana a nuestro punto de vista.

Un caso es la descripción de una situación real, adoptada para estudiarla y propiciar el intercambio de ideas. A pesar de ser detallada, relevante y exhaustiva la descripción del caso no implica que presente todos los acontecimientos que involucran un determinado problema; aunque el caso debe presentarse con claridad y facilitar y permitir el análisis posterior. En muchas 
disciplinas universitarias "[...] los casos son preferidos porque sirven para ilustrar los principios generales y las buenas prácticas, correctas respuestas y hechos que tienen una alta prioridad" (Herreid, 1997: 92).

Los casos son una forma de proporcionar campos de práctica en la formación del estudiante y también del docente porque sitúan al estudiante en una serie de problemas e incluyen prácticas que encontrarán en el mundo real (Roper \& Millar, 1999); el método ayuda a la resolución de auténticos problemas y permite el desarrollo del pensamiento crítico (Mitchem et al., 2008).

Este método es aplicado primordialmente en la enseñanza universitaria, los objetivos y beneficios que pueden obtenerse de él son los siguientes:

- Entendimiento e interpretación de los datos.

- Entendimiento y reconocimiento de suposiciones e inferencias opuestas a lo concreto.

- Hechos.

- Pensamiento analítico y crítico.

- Entendimiento y evaluación de las relaciones interpersonales.

- Ejercitación y elaboración de juicios.

- Comunicación de ideas y opiniones.

- Toma y defensa de decisiones (Roper \& Millar, 1999).

El método proporciona una experiencia sujeta a la realidad y es muy versátil porque permite aplicar proposiciones teóricas y conjugarlas con simulaciones prácticas, además de estimular la creatividad y toma de decisiones. También evita en lo posible la creación de un medio estático, y más bien busca la flexibilidad y la apertura, fomenta la discusión concienzuda del problema de estudio y propicia el intercambio de ideas.

\section{Desarrollo del método de casos}

El método de casos consiste precisamente en proporcionar una serie de casos que representen situaciones problemáticas diversas de la vida real para que se estudien y analicen; asimismo, permite una interrelación fluida de los grupos. El método se estructura estableciendo una secuencia que consideramos necesaria para su desarrollo, se consideran cuatro pasos y se explican de forma sucinta en el Gráfico 1. 


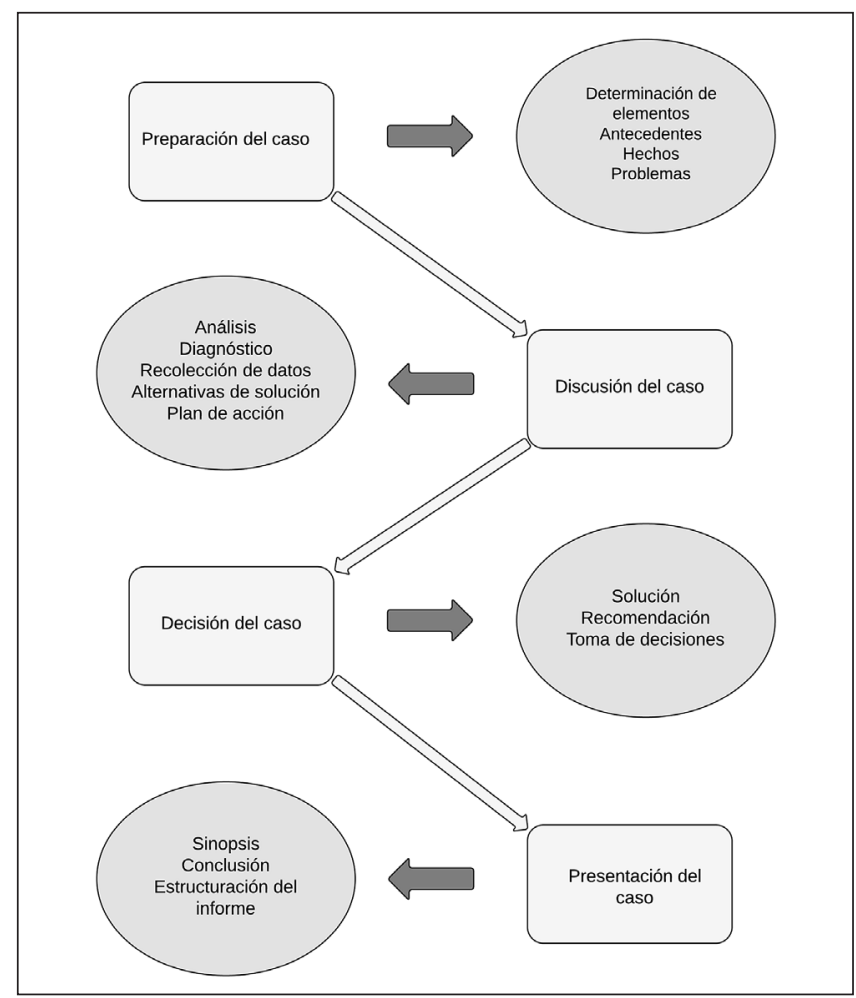

Gráfico 1. Pasos para la aplicación del método de casos.

Fuente: Elaboración propia

Con el método de casos el docente juega un papel muy importante porque queda bajo su responsabilidad el diseño del caso, la preparación de una clase, la pedagogía empleada y la conducción de la clase. Charan (1976) establece que la enseñanza del método de caso es continua y que el instructor (docente) cumple varios roles; puede ser absorbido como iniciador, director, participante y motivador, aunque adicionalmente podríamos añadir el rol de mediador o facilitador.

La presentación del caso, según Gill (2011), requiere de algunas condiciones: realizar una discusión como parte integral del método; utilizar (el facilitador) distintas herramientas para dirigir la discusión; recibir contribuciones de pares; considerar una gran diversidad de las características individuales de los estudiantes; fortalecer las relaciones individuales de los estudiantes, situación importante en la dinámica; reconocer los casos como incompletos; suponer que no hay una única decisión correcta y que la evidencia que apoya una decisión particular puede provenir de muchas fuentes. 
Herreid (1997/1998) advierte que un buen caso cuenta una historia; tiene sumo interés; considera la actualidad (últimos cinco años); genera empatía con los personajes centrales e incluye citas; implica relevancia para el participante, tiene utilidad pedagógica, provoca conflicto, obliga a tomar una decisión y tiene un carácter general. Pero Greenhalgh (2007) va más allá, dice que el método de casos es una ciencia y un arte; reconoce dimensiones científicas y esencialmente literarias desde el punto de vista narrativo.

Hay siete pasos necesarios para adoptar buenas prácticas en la aplicación de este método: mejorar el contacto entre estudiantes y docentes; desarrollar reciprocidad y cooperación entre estudiantes; proporcionar retroalimentación inmediata; priorizar el tiempo en la tarea; generar altas expectativas (con respecto a la solución) y respetar la diversidad de los talentos y formas de aprendizaje. El método presenta algunas complicaciones para quienes poseen dificultades de comunicación o para quienes no responden rápidamente a los problemas presentados (Watson \& Sutton, 2012).

En consecuencia, el método de casos aplicado en la clase tiene un valor fundamental y según la complejidad del mismo puede conducir al estudiante a desarrollar actividades, habilidades, pensamiento crítico e investigación científica (Gráfico 2).

El método de casos y la investigación científica comparten elementos comunes, principalmente el intento general de presentar una situación del mundo real. Hay algunos procedimientos a seguir: los criterios para la selección de los sujetos de la investigación, el establecimiento de la cantidad de casos, la recopilación de la evidencia de la investigación y el análisis y la presentación de los hallazgos (Štrach \& André, 2008).

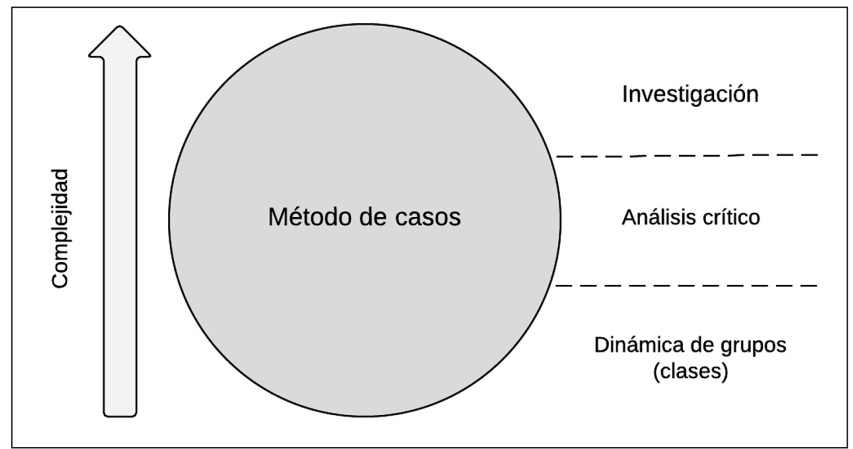

Gráfico 2. Validez pedagógica del método de casos Fuente: Elaboración propia 


\section{Tipología del caso}

Existen diversas propuestas para establecer una tipología de los casos; incluiremos algunas proposiciones que consideramos importantes. Van der Blonk (2003) señala que hay dos dimensiones para escribir un caso. La primera dimensión es definida como reducción-complejidad y la segunda es llamada monólogo-multílogo.

En la dimensión reducción-complejidad, la complejidad está referida al nivel de dificultad que el investigador quiere mostrar acerca del caso; esta dimensión puede incluir material contradictorio, situaciones caóticas, posiciones extremas y emociones. La reducción aminora la riqueza y variedad del caso por un formato predefinido (respuestas claras).

En la dimensión monólogo-multílogo, el caso puede expresar algunos caracteres de una posición determinada y opiniones y puntos de vista propios. La posición del monólogo refleja una historia uniforme y conduce a un solo camino: el camino del autor del caso. La posición multílogo está representada por una interacción de múltiples voces (diferentes posiciones u opiniones), incluida la del autor.

Otra tipología considera nueve tipos de casos: el Caso Iceberg presenta poca información, una estructura pequeña y requiere información adicional, utiliza uno o más modelos conceptuales; el Caso Incidente se encuentra usualmente al final de los capítulos de los libros de texto y presenta incidentes simples y detalles específicos; el Caso Ilustrativo describe factualmente procesos o eventos, la información es abundante e ilustra intensamente la estructura de la situación; en el Caso Central uno o más actores principales interactúan, reflexionan y sus sentimientos son descritos, mientras que la información es provista en alta cantidad y la estructura es débil o floja; el Caso Diálogo es un tipo de caso que promueve la interacción entre uno o más individuos, donde la información provista es moderada y la estructuración baja; el Caso Aplicación describe una técnica o situaciones donde el estudiante requiere de conocimientos técnicos, posee mucha información, pero es altamente desestructurado; el Caso Dato proporciona mucha información y una estructura moderada, aunque la información con que se cuenta resulta irrelevante y es necesario organizar las ideas y el significado de los datos; el Caso Asunto trata un hecho o asunto en cuestión, y usualmente provee mucha información y una estructura moderada; el Caso Predicción implica casos típicamente escritos en una serie y elige un formato para la estructura del caso: la primera parte responde a predicciones correctas y la segunda debe corroborar la predicción (Lundberg et al., 2001). 


\section{Preparación y redacción del caso}

La preparación del caso probablemente requiere de un gran esfuerzo cognoscitivo, Foran (2002) sostiene que el caso debería entenderse de forma rápida y mejorar ostensiblemente la capacidad de participación. El caso necesita de mucha claridad para la posible identificación del problema; los contenidos del caso deben conducir de forma explícita hacia una decisión correcta y posibilitar un rango de soluciones alternativas. Las fuentes para elegir o desarrollar el caso surgen de diversos medios: noticias, Internet, revistas académicas, redes sociales, docentes y estudiantes; entre otros.

La presentación del caso, según Herreid (2011), tiene algunas formas determinadas: método de explicación (narración o argumentación); método de discusión, método de pequeños grupos (para incentivar la discusión, colaboración, integración y participación en clase); casos individuales (no todos los casos son grupales); simulación de casos por computadora; casos clicker donde la utilización de tecnología puede conllevar la discusión académica a mega-aulas o grandes auditorios virtuales y esto permita a los estudiantes elegir entre una variedad de métodos de comunicación que les ayudará a seleccionar un método de acuerdo con su estilo personal de aprendizaje.

Según Wallace (2001) el método del caso evoluciona, porque incorpora los nuevos medios y nuevas las formas de tecnología (multimedia) para promover la formación del docente y del estudiante; se pueden realizar con él interesantes combinaciones de tecnología.

Zimmerman (2002) hace precisiones sobre el modelo clásico del caso y toma en consideración cuatro pasos esenciales: 1) dar preferencia a un caso real con posibles alternativas y resultados; 2 ) proveer información suficiente sobre el caso para su comprensión integral;3) asignar lecturas de fondo prioritarias para la clase; 4) facilitar la discusión en clase, analizar el contenido del caso y elaborar sus posibles soluciones.

A partir de otras conceptualizaciones sobre la estructuración formal del caso, desde nuestro punto de vista, se deberían cumplir seis elementos o pasos fundamentales: 1) Verosimilitud, que sea creíble para el lector; 2) Veracidad, que esté basado en hechos reales; 3 ) Interés y actualidad, que sea novedoso para los lectores; 4) Controversial; que genere discusión; 5) Concreción, que determine los hechos someramente; 6) Comprensión, la complejidad no es sinónimo de confusión.

Corish (2004) muestra otras condiciones útiles para escribir un caso, él considera que deben tomarse en cuenta tres tipos de preguntas que permitan establecer criterios de valor acerca de los conocimientos obtenidos por los estudiantes: Caso Tipo A, preguntas obvias y áreas claramente señaladas en 
el caso. Caso Tipo B, preguntas menos obvias y áreas claramente señaladas y definidas en el caso. Caso Tipo $C$, preguntas y áreas menos obvias en el caso en estudio; esta última condición es usada para ubicar a los mejores candidatos o estudiantes.

Para la redacción de un caso se debería incluir necesariamente un párrafo inicial que dé una visión general del problema, situación o dilema; los antecedentes relevantes y un párrafo final, si el caso lo amerita deberían incluirse anexos o material adicional para su mejor comprensión.

Es necesario tomar en cuenta algunas consideraciones para hacer la descripción del caso, especialmente el autor o investigador debe percibir su rol y posición propia. Wallace (2001) concibe roles en la presentación del caso: uno primario, que cuenta con la colaboración del propio participante quien determina la complejidad; uno secundario donde el participante interpreta el caso según su experiencia; y uno terciario que presenta el caso como un acto interpretativo sin priorizar el conocimiento sino la discusión y la exploración del participante. Además, el autor debe decidir si la estructura del caso es lineal, multilineal o no lineal (Van der Blonk, 2003).

Para evitar algunos problemas respecto de la oportunidad en la presentación del caso es necesario ponerlo a prueba y validarlo con un grupo experimental determinado y así detectar y depurar los posibles errores.

\section{Discusión, decisión y presentación del caso}

Conviene destacar que en el análisis y solución de un caso debe propiciarse un ambiente que permita la discusión y toma de decisiones acerca de las acciones más apropiadas que hay que emprender y orientar hacia la solución del problema que se estudia.

La presentación es un elemento formal que creemos tan importante como la solución de un caso; este aspecto de la presentación de un caso y la resolución del mismo deben elaborarse juntos. Aquí presentamos una posible propuesta para presentar la solución del caso:

- Establecer los antecedentes o resumen de los hechos importantes que pueden determinar las características del caso.

- Determinar el problema, es necesario precisar el problema o problemas principales del caso, realizar una descripción breve y descomponer los elementos del problema.

- Realizar el análisis o diagnóstico, hay que estipular alternativas viables de solución para el problema principal y los secundarios, establecer las 
ventajas y desventajas de las alternativas y el estado del arte del problema, especificando los aspectos técnicos y humanos.

- Decisión, es la solución escogida elaborada en forma breve y la estrategia de aplicación o instrumentalización a seguir, describe las desventajas y formas de neutralizarlas y propone recomendaciones. Algunos casos incluyen apéndices o anexos para reforzar la decisión tomada.

Para una correcta presentación y solución del caso deben evitarse detalles innecesarios, dar tratamiento homogéneo a los distintos hechos y deben considerarse las perspectivas del autor o del curso (Gini, 1985).

\section{Estudio DE CAMPo y APLICACIÓN DEL MÉTODO DE CASOS}

El estudio de campo mostrará la utilización del método y permitirá determinar el nivel de conocimientos adquiridos (conceptos teóricos y técnicas), y también evidenciará mejoras en las relaciones interpersonales, las habilidades, la comunicación, el análisis, la crítica y el uso de información y el manejo de estrategias para el desarrollo del caso.

En la Escuela de Bibliotecología y Ciencias de la Información de la Universidad Nacional Mayor de San Marcos se aplica el método de casos en algunas materias, especialmente en el curso de Derecho y Ciencias de la Información (UNMSM, 2013), y se demostró que la adquisición de conocimientos era positiva y mejor que la enseñanza que aplica otro tipo de evaluaciones. Los estudiantes registraron mejores notas debido al análisis que promueve el caso, en especial en campos como la legislación, los aspectos jurídicos y la ética de la información que son difíciles de internalizar mediante lecturas o disertaciones de los docentes.

Se incluye ahora un estudio de campo en el área de Gestión de la Información, porque se considera que es un área que tiene un número importante de cursos y que tiene preponderancia en el plan de estudios actual.

\section{Objetivos}

Objetivo general

- Demostrar la validez del método de casos en la enseñanza universitaria y en específico en la Bibliotecología y Ciencia de la Información. 
Objetivos específicos

- Conceptualizar y sistematizar el desarrollo del método casos en la enseñanza universitaria.

- Aplicar un estudio de campo utilizando el método de casos con estudiantes de un curso universitario impartido en la Escuela de Bibliotecología y Ciencias de la Información de la Universidad Nacional Mayor de San Marcos.

\section{Construcción y selección del caso}

El caso se confeccionó en función al tema Comportamiento organizacional, tópico 1 y 2 del sílabo del curso Administración de Unidades de Información II del año lectivo 2013. Se trata de involucrar la parte cognitiva, procedimental y actitudinal; se abarca implícitamente el tema de liderazgo, motivación, trabajo en equipo, toma de decisiones, así como la aplicación de herramientas de gestión y herramientas de diagnóstico; no se deja de lado la parte de actitudes y valores. En concreto el caso busca la resolución de problemas, el pensamiento analítico y las habilidades para tomar decisiones en la gestión.

Para la elaboración del caso se tomaron notas de la enseñanza y se agruparon conceptos y temas que serían debatidos en el desarrollo de la clase. Una vez que se recogieron estos datos se incorporaron al caso y posteriormente se le dio forma a una historia con las categorías o temas seleccionados (ver Anexo 1).

\section{Validación del caso}

La validación se hizo a través del juicio de expertos (docentes del área de gestión de la Escuela de Bibliotecología), que ayudaron a evaluar los ítems seleccionados e incorporados en el caso, y también en el test, de forma cuantitativa (pertinencia) y cualitativa (claridad y suficiencia).

Se evaluaron la validez de contenido y del constructo. La validez del contenido (consistencia y relevancia en la elaboración del caso) buscó incluir sólo los aspectos seleccionados para el análisis de los estudiantes, mientras que la validez del constructo fue necesaria para los instrumentos de medición. El estudio utilizó la prueba no paramétrica U de Mann-Whitney para el pretest y el postest aplicados a los estudiantes.

\section{Tipo de caso}

Por un lado se tomó como referencia la tipología de Van der Blonk (2003), que considera que se trata de un caso de reducción-complejidad, pues invo- 
lucra situaciones caóticas, posiciones extremas y, sobretodo, emociones. Por otro lado, según propuesta de Lundberg et al. (2001) es un Caso Diálogo porque promueve la interacción entre uno o más individuos y la información provista es moderada y estructurada.

\section{Metodología}

Para la realización del presente trabajo se aplicó la técnica de la revisión documental para tratar de demostrar la validez del método de casos en la enseñanza y se revisaron las características, procedimientos, tipología y elementos necesarios para la correcta utilización de este método y técnica. La investigación es de carácter experimental, en la modalidad cuasiexperimental, tipo aplicada y de nivel explicativa. Los datos se obtuvieron de una misma prueba escrita -pretest y postest- aplicada a estudiantes de Bibliotecología y Ciencias de la Información de la Universidad Nacional Mayor de San Marcos del sexto semestre, a quienes se les impartía el curso académico Administración de Unidades de Información II del año lectivo 2013. El contenido considerado en el estudio es el tema Comportamiento organizacional y el universo de la muestra fue de 30 estudiantes. A 15 de ellos se les aplicó el método de casos y a los otros 15 se les aplicó la metodología tradicional de exposición de contenidos. Para el análisis de los datos se aplicó la prueba no paramétrica U de Mann-Whitney. Esta prueba es una herramienta estadística útil para detectar diferencias entre dos muestras y es utilizada, por ejemplo, para analizar y comparar datos informétricos (Huber \& Wagner-Döbler, 2003); para comparar la percepción de grupos universitarios diversos acerca de la calidad de los servicios de biblioteca (Shoeb \& Ahmed, 2009) o para contrastar las preferencias de la formación tradicional y la formación en línea junto a otras variables demográficas (Haley, 2008).

\section{Resultados}

En la Tabla 1 se presentan los resultados del pretest (prueba escrita utilizando contenidos de comportamiento organizacional), se observa que los estudiantes del grupo de control y del experimental tienen similares resultados, lo que garantiza situaciones similares al inicio del experimento. En el postest se observa que los resultados del grupo de control y del experimental mejoran significativamente, ya que en ambos grupos ha existido una intervención de mejora; sin embargo, los resultados más significativos se dan en el grupo 
experimental, que fue el que recibió la enseñanza de contenidos relativos al comportamiento organizacional utilizando el método de casos.

Tabla 1. Resultados de la comparación del pretest y postest de la estrategia de estudio

\begin{tabular}{|c|c|c|c|c|c|}
\hline \multirow[t]{2}{*}{ Categorías } & \multicolumn{4}{|c|}{ Grupo } & \multirow[t]{2}{*}{ Test U de Mann-Whitney } \\
\hline & \multicolumn{2}{|c|}{ Experimental $(n=15)$} & \multicolumn{2}{|c|}{ Control $(n=15)$} & \\
\hline \multicolumn{6}{|c|}{ Pretest } \\
\hline & Casos & $\%$ & Casos & $\%$ & \\
\hline Bajo & 12 & $80 \%$ & 13 & $86.67 \%$ & $U=105$ \\
\hline Medio & 3 & $20 \%$ & 2 & $13.33 \%$ & $Z=-.482$ \\
\hline Alto & 0 & $0 \%$ & 0 & $0 \%$ & $p=.630$ \\
\hline \multicolumn{6}{|c|}{ Postest } \\
\hline & Casos & $\%$ & Casos & $\%$ & \\
\hline Bajo & 0 & $0 \%$ & 0 & $0 \%$ & $U=67.5$ \\
\hline Medio & 3 & $20 \%$ & 9 & $60 \%$ & $Z=-2.198$ \\
\hline Alto & 12 & $80 \%$ & 6 & $40 \%$ & $p=0.028$ \\
\hline
\end{tabular}

Fuente: Elaboración propia

En el pretest se muestra el índice de significancia $(\mathrm{p}=0.63>0.05)$ y en el postest es de $(\mathrm{p}=0.028>0.05)$ lo cual se interpreta como que los resultados del pretest son significativos; sin embargo, esta significancia es mucho más notoria en el postest. Por lo tanto, se corrobora que el método de casos influye significativamente en la enseñanza-aprendizaje, específicamente en el tema de Comportamiento organizacional, contenido utilizado como experimento para el caso del presente estudio.

Asimismo se observa, por los porcentajes obtenidos, que en el aprendizaje representado en los niveles bajo, medio y alto de ambos grupos, el pretest estuvo concentrado significativamente en el nivel bajo (80 \%). En el postest un gran porcentaje obtuvo resultados diferenciados del anterior, mientras que en el grupo de control se concentró en el nivel medio (60%); esto podría ser consecuencia de la aplicación de la metodología de exposición de contenidos (disertación) y definitivamente hubo una mejora, pero en el grupo experimental (el cual recibió la intervención del método de casos) hubo una mejora significativa, ya que la gran mayoría se concentró en el nivel bueno (80 \%), lo que nos confirma la efectividad de esta alternativa pedagógica.

Como ha quedado demostrado, desde el punto de vista cuantitativo esta metodología mejora significativamente el aprendizaje; sin embargo, lo más enriquecedor de la experiencia es demostrar las mejoras desde el punto de vista cualitativo, ya que después de la experiencia se observó lo siguiente: 
mayor motivación y participación del estudiante, posición más crítica ante los hechos, mayor compromiso, clima más agradable de aprendizaje en el aula, mayor interacción entre estudiantes, mejora en el nivel de conocimientos y, lo más importante, mejora en la comunicación docente-estudiante.

\section{Conclusiones}

1. El método de casos mejoró significativamente el aprendizaje de los contenidos del curso de Administración de Unidades de Información, presentando en el postest un $\mathrm{p}=0.028>0.05$, lo que se interpreta como una mejora muy significativa.

2. Además de las mejoras cuantitativas, se pudieron evidenciar otros beneficios para el aprendizaje con la utilización de esta metodología, los que se pueden sintetizar en mayor participación del estudiante, mayor interacción y, sobre todo, en que se logran climas más adecuados y agradables para el aprendizaje.

3. Este método tiene un valor indiscutible en la enseñanza académica universitaria actual, especialmente en la Bibliotecología y Ciencias de la Información, pues permite el desarrollo del pensamiento crítico del estudiante y lo prepara para situaciones reales que se presentarán en su futura experiencia profesional.

4. El método tiene múltiples formas de presentación o aplicación pero debe cumplir con algunos requisitos indispensables para generar interés: debe ser verosímil, real, controversial, comprensible, concreto, claro y tener niveles de complejidad.

5. Este mismo método puede ayudar a desarrollar muchos cursos en donde los dilemas no pueden ser resueltos unívocamente; también brinda alternativas para una discusión entre diversos puntos de vista $y$, fundamentalmente, proyecta una interacción permanente del profesional (o estudiante) con su entorno personal, académico o laboral.

6. El método de casos asimismo permite, según el experimento realizado, incluir muchos contenidos en muy poco tiempo y garantizar el aprendizaje significativo de los mismos, pues el estudiante aplica los conocimientos asimilados a una situación práctica concreta, al igual que hace un uso eficiente del tiempo y origina clases de estudio más productivas y efectivas. 


\section{REFERENCIAS BIBLIOGRÁFICAS}

Ardalan, K. (2013), "The philosophical foundation of the lecture method of instruction and the case method of instruction: Implications for examinations", en Contemporary Issues in Education Research (Online), 6 (1), pp. 1-8.

Bañares Parera, L. (2011), "Case method as directed learning in professional ethics”, en Interdisciplinary Studies Journal, 1 (3), pp. 4752.

Corish, A. (2004), "Case studies and examinations", en Teaching Business E Economics, 8 (3), pp. 25-27.

Charan, R. (1976), "Classroom techniques in teaching by the case method", en Academy of Management. The Academy of Management Review, 1 (3), p. 116.

Foran, J. (2002), Case method: Student guidelines. Disponible en: http://www.soc.ucsb.edu/projects/casemethod/guidelines.html

Garvin, D. A. (2003), "Making the Case", en Harvard Magazine, 106 (1), pp. 56-65.

Gill, T. G. (2011), "Informing with the case method", 551 pp. Disponible en: http://www.grandon.com/publications/CaseMethod.pdf

Gini, A. R. (1985), “The case method: A perspective”, en Journal of Business Ethics, 4 (4), pp. 351-352. DOI: 10.1007/bf00381777

Greenhalgh, A. M. (2007), "Case method teaching as science and art: A metaphoric approach and curricular application", en Journal of Management Education, 31 (2), pp. 181-194.

Haley, C. K. (2008), “Online Workplace Training in Libraries”, en Information Technology and Libraries, 27 (1), pp. 33-40.

Hannabuss, S. (1996), "Teaching library and information ethics", en Library Management, 17 (2), pp. 24-35.

Herreid, C. F. (1997), "What is a case: Bringing to Science Education the established teaching tool of Law and Medicine", en Journal of College Science Teaching, 27 (2), pp. 92-94

(1997/1998), "What makes a good case?: Some basic rules of good storytelling help teachers generate student excitement in the classroom", en Journal of College Science Teaching, 27 (3), pp. 163165.

(2011), "Case study teaching", en New Directions for Teaching and Learning, 2011 (128), pp. 31-40. DOI: 10.1002/tl.466

Huber, J. C. \& Wagner-Döbler, R. (2003), "Using the Mann-Whitney test on informetric data", en Journal of the American Society for Information Science and Technology, 54 (8), pp. 798-801. DOI: 10.1002 /asi.10252

Lundberg, C. C.; Rainsford, P.; Shay, J. P. \& Young, C. A. (2001), "Case writing reconsidered", en Journal of Management Education, 25 (4), pp. 450-463. 
Mitchem, K.; Fitzgerald, G.; Hollingsead, C.; Koury, K.; Miller, K. \& Tsai, H.-H. (2008), "Enhancing case-based learning in teacher Education through online discussions: Structure and facilitation", en Journal of Interactive Learning Research, 19 (2), pp. 331-349.

RAE (2013), Diccionario de la lengua española, $22^{\circ}$ ed. Disponible en: http://lema.rae.es/drae/

Roper, M. \& Millar, L. (1999), Writing case studies: A manual. Disponible en: www.irmt.org/documents/educ_training/educ_reso urce/IRMT_ed_rec_writing_cs.doc

Shoeb, Z. H. \& Ahmed, Z. (2009), "Individual differences in service quality assessment: A study of a private university library system in Bangladesh", en Performance Measurement and Metrics, 10 (3), pp. 193-211.

Štrach, P. \& André, M. E. (2008), “Transforming research case studies into teaching cases", en Qualitative Research in Organizations and Management, 3 (3), pp. 199-214. DOI: http://dx.doi.org/10. 1108/17465640810920287

UNMSM (Universidad Nacional Mayor de San Marcos) (2013), Derecho a la información y deontología. Disponible en: http://derecho-informacion-deontologia.blogspot.com/

Van der Blonk, H. (2003), "Writing case studies in information systems research", en Journal of Information Technology, 18 (1), pp. 45-52.

Wallace, J. (2001), "Introduction: Science teaching cases as learning opportunities", en Research in Science Education, 31 (2), pp. 185190. DOI: $10.1023 / \mathrm{a}: 1013126031853$

Watson, S. \& Sutton, J. M. (2012), "An examination of the effectiveness of case method teaching online: Does the technology matter?", en Journal of Management Education, 36 (6), pp. 802-821.

Zimmerman, A. (2002), "Flexibility and variety in the use of case studies”, en NACTA Journal, 46 (3), p. 34 
Anexo 1

\section{Universidad Nacional Mayor de San Marcos \\ Facultad de Letras y Ciencias Humanas \\ EAP. Bibliotecología y CC.II.}

Asignatura: Administración de Unidades de Información II

Apellidos Y Nombres:

Indicaciones:

Con el presente caso se pretende tratar de manera transversal diferentes temas de Comportamiento organizacional, como son: liderazgo, motivación, trabajo de equipo, toma de decisiones, elaboración de herramientas de gestión y de diagnóstico, así como desarrollar la parte actitudinal y valorativa de la gestión de unidades de información. Es necesario trabajarlo grupalmente y ponerse en la situación en mención. Considere que en un caso no hay respuesta correcta o incorrecta. Es necesario sustentar su posición.

Caso de Estudio

"Tema: Comportamiento Organizacional"

Elena es una bachiller en Bibliotecología, recién egresada de la UNMSM, actualmente trabaja como apoyo en un Centro de Documentación donde gana 600 soles mensuales; al revisar una lista de interés llamada Biblio encuentra una oportunidad laboral como Jefa de Biblioteca de la Oficina Nacional de Pensiones, ganará un sueldo mayor que fluctúa entre 4,800 y 6,000 soles mensuales. Elena se presenta a la entrevista de trabajo y le otorgan una semana de periodo de prueba, en ese tiempo ella tiene que demostrar que cuenta con los conocimientos y actitudes necesarias para el puesto. Pero encuentra la siguiente realidad en el puesto de trabajo:

- El jefe anterior se fue de muy malas formas por un problema político, y no le ha dejado nada de información sobre la gestión, ni un inventario, ni siquiera el correlativo de oficios necesarios para seguir con la comunicación institucional, ni nada. No sabe qué documentos hay pendientes ni en qué etapa se encuentran las actividades, mientras las autoridades le piden resultados casi inmediatos.

- El personal que existe actualmente goza de estabilidad laboral obtenida por intermedio de los sindicatos y tiene poca disposición de ayudar; además de ello están dispuestos a hacer la vida imposible a la nueva jefa, ya que muchos de ellos querían obtener ese puesto.

- Se observan muchas deficiencias en la organización, como: falta de control en los préstamos, el acceso a la colección es indiscriminada, hay desmotivación entre el personal, no hay quien dirija la institución, no hay comunicación interpersonal, no hay planes a corto, mediano o largo plazo, no hay funciones definidas, entre otras cosas.

\section{Si fueses Elena:}

¿Aceptarías el trabajo?

Si fuese así: ¿qué acciones inmediatas tomarías para administrar esta biblioteca?

¿Qué herramientas de diagnóstico y gestión pondrías en práctica? ¿Qué actitudes pondrías en práctica?

Ciudad Universitaria, 27 de agosto de 2013 